\title{
Diagnostic change 10 years after a first episode of psychosis
}

\author{
M. Heslin ${ }^{1} t^{*}$, B. Lomas $^{2}$ t, J. M. Lappin ${ }^{3,4}$, K. Donoghue ${ }^{5}$, U. Reininghaus ${ }^{6,7,8}$, A. Onyejiaka ${ }^{9}$, \\ T. Croudace ${ }^{10}$, P. B. Jones ${ }^{11}$, R. M. Murray ${ }^{4}$, P. Fearon ${ }^{12}$, P. Dazzan ${ }^{4}$, C. Morgan ${ }^{6}$ and G. A. Doody ${ }^{2}$ \\ ${ }^{1}$ Centre for Economics of Mental and Physical Health, King's College London, London, UK \\ ${ }^{2}$ Division of Psychiatry, University of Nottingham, Nottingham, UK \\ ${ }^{3}$ Department of Psychiatry, University of New South Wales, Sydney, Australia \\ ${ }^{4}$ Psychosis Studies Department, King's College London, London, UK \\ ${ }^{5}$ Addictions Department, King's College London, London, UK \\ ${ }^{6}$ Centre for Epidemiology and Public Health, King's College London, London, UK \\ ${ }^{7}$ Department of Psychiatry and Psychology, School for Mental Health and Neuroscience, Maastricht University, Maastricht, The Netherlands \\ ${ }^{8}$ NIHR Collaboration for Leadership in Applied Health Research \& Care, Cambridge, UK \\ ${ }^{9}$ Department of Psychology, King's College London, London, UK \\ ${ }^{10}$ School of Nursing and Midwifery, College of Medicine, Dentistry and Nursing, University of Dundee, Dundee, UK \\ ${ }^{11}$ Department of Psychiatry, University of Cambridge, Cambridge, UK \\ ${ }^{12}$ Department of Psychiatry, Trinity College, Dublin, Republic of Ireland
}

Background. A lack of an aetiologically based nosology classification has contributed to instability in psychiatric diagnoses over time. This study aimed to examine the diagnostic stability of psychosis diagnoses using data from an incidence sample of psychosis cases, followed up after 10 years and to examine those baseline variables which were associated with diagnostic change.

Method. Data were examined from the ÆSOP and ÆSOP-10 studies, an incidence and follow-up study, respectively, of a population-based cohort of first-episode psychosis cases from two sites. Diagnosis was assigned using ICD-10 and DSM-IV-TR. Diagnostic change was examined using prospective and retrospective consistency. Baseline variables associated with change were examined using logistic regression and likelihood ratio tests.

Results. Slightly more (59.6\%) cases had the same baseline and lifetime ICD-10 diagnosis compared with DSM-IV-TR $(55.3 \%)$, but prospective and retrospective consistency was similar. Schizophrenia, psychotic bipolar disorder and drug-induced psychosis were more prospectively consistent than other diagnoses. A substantial number of cases with other diagnoses at baseline (ICD-10, $n=61$; DSM-IV-TR, $n=76$ ) were classified as having schizophrenia at 10 years. Many variables were associated with change to schizophrenia but few with overall change in diagnosis.

Conclusions. Diagnoses other than schizophrenia should to be regarded as potentially provisional.

Received 19 December 2014; Revised 19 March 2015; Accepted 24 March 2015; First published online 4 May 2015

Key words: Diagnosis, first-episode psychosis, psychoses.

\section{Introduction}

Diagnosis in psychiatry has frequently come under fire. Robins \& Guze (1970) discussed how clinical features, outcome and family history can be used to create nosological categories in the absence of clinical tests. The National Institute of Mental Health recently criticized the validity of the Diagnostic and Statistical

\footnotetext{
* Address for correspondence: M. Heslin, Ph.D., Centre for the Economics of Mental and Physical Health, Institute of Psychiatry, Psychology and Neuroscience at King's College London, Box 024, The David Goldberg Centre, De Crespigny Park, Denmark Hill, London SE5 8AF, UK.

(Email: Margaret.Heslin@kcl.ac.uk)

† Joint first authors.
}

Manual of Mental Disorders (DSM), stating 'Unlike our definitions of ischemic heart disease, lymphoma, or AIDS, the DSM diagnoses are based on a consensus about clusters of clinical symptoms, not any objective laboratory measure' (Lane, 2013). This lack of aetiologically based psychiatric classification has contributed to instability in psychiatric diagnoses over time.

A number of studies have examined diagnostic stability over time. Early research was heterogeneous in nature, reporting on differing diagnostic criteria, different diagnostic processes and differing methods of reporting results. More recently, however, researchers have followed the lead of Schwartz et al. (2000) in reporting prospective and retrospective consistencies of diagnoses between two time points. Prospective consistency is the proportion of cases that receives a

This is an Open Access article, distributed under the terms of the Creative Commons Attribution licence (http://creativecommons.org/licenses/by/ $3.0 /$ ), which permits unrestricted re-use, distribution, and reproduction in any medium, provided the original work is properly cited. 
diagnosis at baseline and retains that diagnosis at follow-up. Retrospective consistency is the proportion of cases that receives a diagnosis at follow-up that they also had at baseline.

The prospective consistencies of schizophrenia and bipolar affective disorder have been reported at $80-100 \%$ in studies with up to 4 years of follow-up using first admission or first contact samples and more recent criteria [International Classification of Diseases (ICD)-10 or DSM-III onwards] (Amin et al. 1999; Schwartz et al. 2000; Veen et al. 2004; Baldwin et al. 2005; Whitty et al. 2005; Salvatore et al. 2009, 2011). Studies with shorter follow-up periods report that diagnoses are more stable over time. Longerterm follow-up of the cohorts, identified by the Determinants of Outcome of Severe Mental Disorder (DOSMeD), World Health Organization Collaborative Study on the Assessment and Reduction of Social Disability (RAPyD) and International Pilot Study of Schizophrenia (IPSS) samples reported by Craig et al. (2007) identified prospective consistencies for schizophrenia of about $85 \%$ over $12-26$ years of follow-up, using ICD-10 diagnoses converted using a World Health Organization algorithm from previous classification systems. Bromet et al. (2011) reported on the 10-year follow-up of a first admission cohort and identified similar diagnostic stability findings for schizophrenia and bipolar affective disorder.

Findings with regards to other psychotic categories are more variable. Depressive psychosis prospective consistencies are reported to be between 45 and 55\% (Baca-Garcia et al. 2007a, b; Bromet et al. 2011) in longterm follow-up studies (10-12 years), compared with $75-100 \%$ in shorter-term follow-up studies (1.5-4 years; Schwartz et al. 2000; Schimmelmann et al. 2005; Whitty et al. 2005; Salvatore et al. 2009, 2011). Smaller diagnostic categories (where studied) demonstrate more variation still. Schizo-affective disorder, for example, has reported prospective consistencies ranging from 35\% (Schwartz et al. 2000; Craig et al. 2007) to 95-100\% (Schimmelmann et al. 2005; Salvatore et al. 2011). This variation probably reflects differences in the diagnostic system adopted, cohort (first contact $v$. first hospitalization) and length of follow-up. Further studies have attempted to address stability in less prevalent diagnoses such as acute and transient psychosis. These studies also show considerable variability in prospective consistency: $30 \%$ (Aadamsoo et al. 2011); 73\% (Sajith et al. 2002).

Many studies mentioned above used non-incidence samples. Incidence studies give a less biased estimate of diagnostic stability, as studies that recruit cases from non-first-episode samples are effectively sampling chronic cases in treatment, biasing the investigation towards those who are more unwell and excluding others who recover. One study has examined long-term diagnostic stability (13 years) in an incidence sample of psychosis patients but only including schizophrenia cases (Mason et al. 1997). To date, no study has yet examined diagnostic stability of all psychosis diagnoses specifically in incident cases over a follow-up period of longer than 8 years using current diagnostic criteria.

Identification of predictors of diagnostic change over time is important because predictors may aid in understanding why diagnoses change and indicate to clinical teams when to be attentive to potential change and adjust treatment accordingly. Studies of relatively short duration have examined predictors of diagnostic change in an incidence cohort, but only one study has looked at a long-term follow-up of diagnostic categories, in first admission cases (Schwartz et al. 2000; Salvatore et al. 2009; Bromet et al. 2011).

In this study we sought to complement the knowledge obtained from previous research, using a geographically defined incident sample of all psychoses diagnoses made using DSM-IV-TR and ICD-10 criteria, followed up and rediagnosed 10 years later, to fulfil two main aims: (1) to examine the stability of diagnostic categories 10 years after the first episode of psychosis; and (2) to examine which demographic and clinical characteristics are associated with diagnostic change.

\section{Method}

\section{Baseline}

This paper is based on data from the ÆSOP study (Kirkbride et al. 2006; Morgan et al. 2014), an incidence study of all first-episode psychosis cases presenting for the first time to specialist mental health services in defined catchment areas in Nottingham and London.

At baseline, clinical and demographic data were collected from clinical records and, where possible, from interview with cases using the Schedules for Clinical Assessment in Neuropsychiatry (SCAN version 2; World Health Organization, 1994) and the Personal and Psychiatric History Schedule (World Health Organization, 1996). The SCAN was used to elicit symptom-related data at the time of presentation. Where an interview with the patient was not possible, case notes were used to complete the Item Group Checklist (IGC) part of the SCAN. Case summaries collated all relevant clinical information and were made available to consensus diagnostic meetings alongside the schedules above. ICD-10 (World Health Organization, 1993) and DSM-IV-TR (American Psychiatric Association, 2000) psychotic diagnoses were determined using all available clinical information (excluding clinical diagnosis) on the basis of 
consensus meetings involving at least one of the principal investigators with other members of the research team (at least one psychiatrist and one other member of the research team). Conflicting opinions on diagnosis were discussed in detail until a consensus was reached. This was made as soon as possible after first contact (generally within a few weeks). Cases with a dual diagnosis had their substance use disorder diagnosed separately. Diagnoses were made blind to ethnicity. Factor analyses of the SCAN and IGC data generated the following symptom dimensions: manic; depressive; disorganized; negative; and reality distortion (Demjaha et al. 2009).

\section{Follow-up}

Cases were followed up 10 years after first contact with services in ÆSOP-10 (Morgan et al. 2014). The World Health Organization Life Chart (Susser et al. 2000; Harrison et al. 2001) was completed for each case using case notes and clinical interview where possible, to map course of illness and symptom history. The SCAN was also completed in relation to the preceding month where possible. Lifetime diagnosis (based on information from baseline to follow-up) using a consensus approach was based on all this clinical information, and blind to ethnicity and baseline diagnoses.

Ethical approval was granted by the Institute of Psychiatry and South London and Maudsley (SLaM) Research Ethics Committee and by the North Nottingham Healthcare NHS Trust Ethics Committee.

\section{Analyses}

Data were analysed using STATA 10 (StataCorp, 2009). Differences between followed-up and not-followed-up cases were analysed using $\chi^{2}$ and Wilcoxon rank-sum tests as appropriate. Prospective and retrospective consistencies were calculated as the percentage of cases with the same diagnosis at follow-up as at baseline, and the percentage of cases with the same diagnosis at baseline as at follow-up, respectively.

Predictors of diagnostic change were assessed in two steps: unadjusted univariate logistic regression analyses; and models of independent predictors. Models of independent predictors were built by starting with a single variable and adding in additional variables, one at a time to examine their effect on the model. Only variables with $p<0.1$ in the unadjusted analyses were entered into the adjusted regression model starting with the variable with the biggest effect. Each additional variable was then added to the model in turn using likelihood ratio tests to determine if that variable significantly improved the model or not. Variables that did not improve the model were excluded from the model. Predictors were simplified into binary factors where possible in order to simplify analyses and increase power. As well as analysing age as a continuous variable, it was reclassified into a binary variable (in the age risk period: males under the age of 40 years or females under the age of 50 years) as this has been indicated as an important predictor in the development of schizophrenia (Häfner et al. 1998).

\section{Results \\ Sample}

At baseline, a total of 557 first-episode psychosis cases were identified. Data here are based on the incidence sample collected over the first 2 years (excluding: nonincidence cases collected for the brain imaging component of the study; cases oversampled in the third year in order to increase the numbers for the ethnicity component of the study; and cases excluded post-baseline). This led to a total number of 505 cases: 304 from London and 201 from Nottingham.

Of the 505 patients eligible for follow-up, 79.8\% (403) had sufficient information to make a lifetime diagnosis based on at least 8 years of information (including 33 cases who had died during the follow-up period but had sufficient information to have a lifetime diagnosis made). Therefore, a total of 102 cases had no follow-up diagnostic information. The mean follow-up in years of those who had a lifetime diagnosis and were still alive was 10.74 years (S.D. $=1.17$, range $8.08-13.70$ ).

Table 1 shows the differences in demographic and clinical variables between those with and without a diagnosis at 8 or more years. Centre and place of birth were associated with lifetime diagnosis status. Cases who were born abroad were more likely to move abroad over the follow-up period than cases born in the UK and therefore less likely to be followed up. More cases from London were not born in the UK $(38 \%, 112 / 297)$ compared with Nottingham $(9 \%, 18 / 201$; $p<0.01)$, and so a lower lifetime diagnosis rate in London could be linked to place of birth.

\section{Diagnostic change: prospective and retrospective consistency}

Of cases, 59.6\% (240/403) had the same baseline and lifetime ICD diagnosis, and 55.3\% (223/403) of cases had the same baseline and lifetime DSM diagnosis. A substantial proportion of patients had a baseline and lifetime diagnosis of schizophrenia in both the ICD (33\%) and DSM (28\%) (Tables 2 and 3). The DSM system produced four cases with a baseline or lifetime diagnosis of either 'unknown' or 'other'. This was due to the fact that although there was a large amount of information for 8 or more years, the diagnostic team was unable to agree upon a diagnosis. 
Table 1. Comparison of key variables between those with a diagnosis and those without a diagnosis

\begin{tabular}{|c|c|c|c|c|c|}
\hline & \multirow[b]{2}{*}{$\begin{array}{l}\text { No diagnosis, } \\
n(\%)(n=102)\end{array}$} & \multirow[b]{2}{*}{$\begin{array}{l}\text { Diagnosis, } \\
n(\%)(n=403)\end{array}$} & \multicolumn{2}{|c|}{ Test statistic } & \multirow[b]{2}{*}{$p$} \\
\hline & & & $\chi^{2}(\mathrm{df})$ & $\begin{array}{l}\text { Wilcoxon } \\
\text { Z }\end{array}$ & \\
\hline \multicolumn{6}{|l|}{ Baseline diagnosis } \\
\hline Schizophrenia & $41(18.8)$ & $177(81.2)$ & $2.088(8)$ & & 0.973 (Fisher's exact) \\
\hline Delusional disorder & $3(13.6)$ & $19(86.4)$ & & & \\
\hline Acute and transient psychoses & $7(24.1)$ & $22(75.9)$ & & & \\
\hline Schizo-affective & $6(19.4)$ & $25(80.7)$ & & & \\
\hline $\begin{array}{l}\text { Bipolar disorder and mania with } \\
\text { psychotic features }\end{array}$ & $15(21.4)$ & $55(78.6)$ & & & \\
\hline $\begin{array}{l}\text { Major depression with psychotic } \\
\text { symptoms }\end{array}$ & $17(23.6)$ & $55(76.4)$ & & & \\
\hline Schizotypal personality disorder & $0(-)$ & $1(100)$ & & & \\
\hline Drug-induced psychoses & $5(19.2)$ & $21(80.8)$ & & & \\
\hline Psychoses NOS & $8(22.2)$ & $28(77.8)$ & & & \\
\hline \multicolumn{6}{|l|}{ Grouped diagnoses } \\
\hline Non-affective psychoses & $70(19.2)$ & $293(80.7)$ & $0.7744(2)$ & & 0.679 \\
\hline $\begin{array}{l}\text { Bipolar disorder and mania with } \\
\text { psychotic features }\end{array}$ & $15(21.4)$ & $55(78.6)$ & & & \\
\hline $\begin{array}{l}\text { Major depression with psychotic } \\
\text { symptoms }\end{array}$ & 17 (23.6) & $55(76.4)$ & & & \\
\hline \multicolumn{6}{|l|}{ Centre } \\
\hline London & $73(24.0)$ & $231(76.0)$ & $6.897(1)$ & & 0.009 \\
\hline Nottingham & $29(14.4)$ & $172(85.6)$ & & & \\
\hline \multicolumn{6}{|l|}{ Gender } \\
\hline Male & $63(21.5)$ & $230(78.5)$ & $0.736(1)$ & & 0.391 \\
\hline Female & $39(18.4)$ & $173(81.6)$ & & & \\
\hline \multicolumn{6}{|l|}{ Ethnicity } \\
\hline White British & $43(18.9)$ & $185(81.1)$ & $10.570(5)$ & & 0.061 \\
\hline African-Caribbean & $18(15.1)$ & $101(84.9)$ & & & \\
\hline Black African & $17(26.2)$ & 48 (73.9) & & & \\
\hline White other & $10(27.8)$ & $26(72.2)$ & & & \\
\hline Asian & $3(11.5)$ & $23(88.5)$ & & & \\
\hline Other & $11(35.5)$ & $20(64.5)$ & & & \\
\hline \multicolumn{6}{|l|}{ Place of birth } \\
\hline Non-UK born & $35(26.9)$ & $95(73.1)$ & $4.800(1)$ & & 0.028 \\
\hline UK born & $66(17.9)$ & $302(82.1)$ & & & \\
\hline Age at first contact, years & & & & -1.617 & 0.106 \\
\hline Median & 27 & 29 & & & \\
\hline IQR & $22-33$ & $22-36$ & & & \\
\hline Age at onset, years & & & & -1.597 & 0.110 \\
\hline Median & 27 & 29.5 & & & \\
\hline IQR & $22-34$ & $23-38$ & & & \\
\hline DUP, days & & & & -0.248 & 0.804 \\
\hline Median & 56 & 60 & & & \\
\hline IQR & $15-184$ & $15-238$ & & & \\
\hline
\end{tabular}

df, Degrees of freedom; NOS, not otherwise specified; IQR, interquartile range; DUP, duration of undiagnosed psychosis.

Table 4 presents the prospective and retrospective consistency of the ICD-10 and DSM-IV-TR. The DSM and ICD had very similar prospective consistencies for most diagnoses, with schizophrenia, psychotic bipolar disorder and drug-induced psychosis having the highest prospective consistency. In terms of retrospective consistency, schizophrenia diagnosed using the DSM had almost 10\% lower consistency than 
Table 2. ICD movement matrix

\begin{tabular}{|c|c|c|c|c|c|c|c|c|c|c|}
\hline \multirow[b]{2}{*}{ Lifetime diagnosis $(n)$} & \multicolumn{10}{|c|}{ Baseline diagnosis $(n)$} \\
\hline & Schizophrenia & $\begin{array}{l}\text { Schizo-affective } \\
\text { disorder }\end{array}$ & $\begin{array}{l}\text { Delusional } \\
\text { disorder }\end{array}$ & $\begin{array}{l}\text { Acute and } \\
\text { transient } \\
\text { psychoses }\end{array}$ & $\begin{array}{l}\text { Bipolar disorder } \\
\text { and mania with } \\
\text { psychotic } \\
\text { features }\end{array}$ & $\begin{array}{l}\text { Major } \\
\text { depression with } \\
\text { psychotic } \\
\text { features }\end{array}$ & $\begin{array}{l}\text { Schizotypal } \\
\text { personality } \\
\text { disorder }\end{array}$ & $\begin{array}{l}\text { Drug-induced } \\
\text { psychoses }\end{array}$ & $\begin{array}{l}\text { Psychosis } \\
\text { NOS }\end{array}$ & Total \\
\hline Schizophrenia & 133 & 8 & 12 & 7 & 2 & 15 & 1 & 3 & 13 & 194 \\
\hline $\begin{array}{l}\text { Schizo-affective } \\
\text { disorder }\end{array}$ & 16 & 9 & 0 & 0 & 3 & 3 & 0 & 1 & 5 & 37 \\
\hline Delusional disorder & 0 & 0 & 3 & 1 & 0 & 2 & 0 & 0 & 0 & 6 \\
\hline $\begin{array}{l}\text { Acute and transient } \\
\text { psychoses }\end{array}$ & 1 & 1 & 0 & 4 & 2 & 1 & 0 & 1 & 0 & 10 \\
\hline $\begin{array}{l}\text { Bipolar disorder and } \\
\text { mania with } \\
\text { psychotic features }\end{array}$ & 7 & 3 & 0 & 1 & 42 & 7 & 0 & 0 & 1 & 61 \\
\hline $\begin{array}{l}\text { Major depression } \\
\text { with psychotic } \\
\text { features }\end{array}$ & 8 & 1 & 1 & 5 & 2 & 26 & 0 & 0 & 1 & 44 \\
\hline $\begin{array}{l}\text { Schizotypal } \\
\text { personality disorder }\end{array}$ & 0 & 0 & 0 & 0 & 0 & 0 & 0 & 0 & 1 & 1 \\
\hline $\begin{array}{l}\text { Drug-induced } \\
\text { psychoses }\end{array}$ & 5 & 1 & 0 & 5 & 2 & 0 & 0 & 16 & 0 & 29 \\
\hline Psychoses NOS & 7 & 2 & 1 & 1 & 2 & 1 & 0 & 0 & 7 & 21 \\
\hline Total & 177 & 25 & 17 & 24 & 55 & 55 & 1 & 21 & 28 & 403 \\
\hline
\end{tabular}

ICD, International Classification of Diseases; NOS, not otherwise specified. 
Table 3. DSM movement matrix

\begin{tabular}{|c|c|c|c|c|c|c|c|c|c|c|c|c|}
\hline \multirow[b]{2}{*}{$\begin{array}{l}\text { Lifetime } \\
\text { diagnosis }(n)\end{array}$} & \multicolumn{12}{|c|}{ Baseline diagnosis $(n)$} \\
\hline & Schizophrenia & $\begin{array}{l}\text { Schizo-affective } \\
\text { disorder }\end{array}$ & $\begin{array}{l}\text { Major } \\
\text { depression } \\
\text { with } \\
\text { psychotic } \\
\text { features }\end{array}$ & $\begin{array}{l}\text { Bipolar } \\
\text { disorder } \\
\text { and } \\
\text { mania } \\
\text { with } \\
\text { psychotic } \\
\text { features }\end{array}$ & $\begin{array}{l}\text { Delusional } \\
\text { disorder }\end{array}$ & $\begin{array}{l}\text { Psychosis } \\
\text { NOS }\end{array}$ & Schizophreniform & $\begin{array}{l}\text { Drug/ } \\
\text { alcohol-induced } \\
\text { psychosis }\end{array}$ & $\begin{array}{l}\text { Other } \\
\text { disorder }\end{array}$ & Unclear & $\begin{array}{l}\text { Brief } \\
\text { psychotic } \\
\text { disorder }\end{array}$ & Total \\
\hline Schizophrenia & 113 & 8 & 15 & 2 & 12 & 13 & 17 & 4 & 1 & 1 & 3 & 189 \\
\hline $\begin{array}{l}\text { Schizo-affective } \\
\text { disorder }\end{array}$ & 13 & 6 & 4 & 3 & 0 & 5 & 1 & 0 & 0 & 0 & 0 & 32 \\
\hline $\begin{array}{l}\text { Major depression } \\
\text { with psychotic } \\
\text { features }\end{array}$ & 7 & 1 & 28 & 2 & 1 & 1 & 1 & 0 & 0 & 0 & 3 & 44 \\
\hline $\begin{array}{l}\text { Bipolar disorder } \\
\text { and mania with } \\
\text { psychotic } \\
\text { features }\end{array}$ & 7 & 2 & 9 & 42 & 0 & 1 & 0 & 0 & 0 & 0 & 1 & 62 \\
\hline $\begin{array}{l}\text { Delusional } \\
\text { disorder }\end{array}$ & 0 & 0 & 2 & 0 & 4 & 0 & 0 & 0 & 0 & 0 & 0 & 6 \\
\hline Psychosis NOS & 6 & 3 & 1 & 3 & 1 & 8 & 1 & 0 & 0 & 0 & 1 & 24 \\
\hline Schizophreniform & 3 & 0 & 0 & 0 & 1 & 1 & 3 & 0 & 0 & 0 & 0 & 8 \\
\hline $\begin{array}{l}\text { Drug / alcohol } \\
\text { induced } \\
\text { psychosis }\end{array}$ & 4 & 1 & 0 & 2 & 2 & 1 & 2 & 16 & 0 & 0 & 1 & 29 \\
\hline Other disorder & 1 & 0 & 0 & 0 & 0 & 0 & 0 & 0 & 0 & 0 & 0 & 1 \\
\hline Unclear & 0 & 0 & 0 & 0 & 0 & 0 & 0 & 0 & 0 & 0 & 1 & 1 \\
\hline $\begin{array}{l}\text { Brief psychotic } \\
\text { disorder }\end{array}$ & 1 & 0 & 1 & 1 & 0 & 0 & 0 & 1 & 0 & 0 & 3 & 7 \\
\hline Total & 155 & 21 & 60 & 55 & 21 & 30 & 25 & 21 & 1 & 1 & 13 & 403 \\
\hline
\end{tabular}

DSM, Diagnostic and Statistical Manual of Mental Disorders; NOS, not otherwise specified. 
Table 4. Prospective and retrospective consistency

\begin{tabular}{|c|c|c|c|c|}
\hline \multirow[b]{2}{*}{ Diagnosis } & \multicolumn{2}{|c|}{$\begin{array}{l}\text { Prospective consistency, \% } \\
\text { (baseline number) }\end{array}$} & \multicolumn{2}{|c|}{$\begin{array}{l}\text { Retrospective consistency, \% } \\
\text { (follow-up number) }\end{array}$} \\
\hline & DSM & ICD & DSM & ICD \\
\hline Schizophrenia & $72.9(155)$ & $75.1(177)$ & $59.8(189)$ & $68.6(194)$ \\
\hline Bipolar disorder and mania with psychotic features & $76.4(55)$ & $76.4(55)$ & $67.7(62)$ & $68.9(61)$ \\
\hline Major depression with psychotic features & $46.7(60)$ & $47.3(55)$ & $63.6(44)$ & $59.1(44)$ \\
\hline Schizo-affective disorder & $28.6(21)$ & $36.0(25)$ & $18.8(32)$ & $24.3(37)$ \\
\hline Delusional disorder & $19.0(21)$ & $17.6(17)$ & $66.7(6)$ & $50.0(6)$ \\
\hline Drug-induced psychoses & $76.2(21)$ & $76.2(21)$ & $55.2(29)$ & $55.2(29)$ \\
\hline Psychoses NOS & $26.7(30)$ & $25.0(28)$ & $33.3(24)$ & $33.3(21)$ \\
\hline Schizophreniform & $12.0(25)$ & - & $37.5(8)$ & - \\
\hline Brief/acute and transient psychoses & $23.1(13)$ & $16.7(24)$ & $37.5(7)$ & $40.0(10)$ \\
\hline
\end{tabular}

DSM, Diagnostic and Statistical Manual of Mental Disorders; ICD, International Classification of Diseases; NOS, not otherwise specified.

schizophrenia diagnosed using the ICD. This difference is accounted for by the use of schizophreniform disorder in the DSM but not in the ICD. Drug-induced psychosis had a marginally higher prospective consistency compared with schizophrenia when using both the ICD and DSM. There were more cases at follow-up than at baseline with the following diagnoses: schizophrenia, schizo-affective disorder, bipolar disorder and drug-induced psychosis.

\section{Predictors of change}

The unadjusted regression analyses for key baseline demographic, clinical and social variables revealed that five baseline variables were associated with change in ICD diagnosis (see online Supplementary Appendix S1 for full details). Likelihood ratio tests revealed that the variables which together created the most parsimonious model associated with change were: having a diagnosis of delusional disorder [odds ratio (OR) 23.42, 95\% confidence interval (CI) 4.15-132.03], acute and transient psychosis (OR 73.84, 95\% CI 8.52-639.80), schizo-affective disorder (OR 9.00, 95\% CI 2.33-34.71) or psychosis not otherwise specified (NOS) (OR 12.74, 95\% CI 2.24-72.39); being from London (Nottingham: OR 0.50, 95\% CI $0.25-1.04$ ); and having depressive symptoms (OR 1.92, 95\% CI 1.11-3.32).

Eight baseline variables were associated with change in DSM diagnosis in the unadjusted analyses. Likelihood ratio tests revealed that the variables which together created the most parsimonious model of change were: having a diagnosis of psychotic major depression (OR 2.54, 95\% CI 0.87-7.41), schizoaffective disorder (OR 12.21, 95\% CI 2.16-69.00), delusional disorder (OR 40.02, 95\% CI 6.39-250.73), psychosis NOS (OR 22.95, 95\% CI 3.88-135.76) and brief psychotic disorder (OR 39.38, 95\% CI 3.53-439.13); being from London (Nottingham: OR 0.46, 95\% CI 0.22-0.98); having a lower age at onset (OR 0.17, 95\% CI 0.05-0.54); having contact with friends (less than weekly contact with friends: OR $0.34,95 \%$ CI $0.13-0.90)$; and having depressive symptoms (OR 2.35, 95\% CI 1.30-4.26).

\section{Predictors of change to schizophrenia}

Considering the high number of cases moving to a diagnosis of schizophrenia over the follow-up period and the implied increase in need this may engender (Carr et al. 2004), a separate analysis of predictors of change to a diagnosis of schizophrenia was undertaken. This is shown in Table 5. For the ICD analyses, all diagnoses were used. However, given the overlap between DSM-IV-TR schizophreniform psychosis and schizophrenia, schizophreniform cases were excluded from the DSM analysis.

The unadjusted analyses revealed that many more variables were associated with ICD and DSM diagnosis change to schizophrenia. For the ICD, there was some evidence that 14 baseline variables were associated with a change to schizophrenia. There was strong evidence that the following were associated with change to schizophrenia: being from London (Nottingham OR 0.31, 95\% CI 0.16-0.58); being in the age risk period (OR 3.43, 95\% CI 1.16-10.14); being black African (OR 3.03, 95\% CI 1.25-7.35); longer duration of undiagnosed psychosis (DUP) (OR 1.16, 95\% CI $0.99-1.35)$; having symptoms of reality distortion (OR 1.73, 95\% CI 1.07-2.81); having negative 
Table 5. Predictors of diagnostic change to schizophrenia using the ICD and DSM

\begin{tabular}{|c|c|c|c|}
\hline Predictor & $\begin{array}{l}\text { ICD unadjusted OR } \\
(95 \% \mathrm{CI})\end{array}$ & Predictor & $\begin{array}{l}\text { DSM unadjusted OR } \\
(95 \% \mathrm{CI})\end{array}$ \\
\hline \multicolumn{4}{|l|}{ Demographics } \\
\hline Centre $(n=226)$ & & Centre $(n=223)$ & \\
\hline London & - & London & - \\
\hline Nottingham & $0.31(0.16-0.58)^{* *}$ & Nottingham & $0.34(0.18-0.65)^{* *}$ \\
\hline Gender $(n=226)$ & & Gender $(n=223)$ & \\
\hline Male & - & Male & - \\
\hline Female & $0.57(0.31-1.03)^{*}$ & Female & $0.50(0.27-0.92)^{* *}$ \\
\hline Log age $(n=226)$ & $0.62(0.25-1.50)$ & Log age $(n=223)$ & $0.49(0.20-1.23)$ \\
\hline In the age risk period $(n=226)$ & & In the age risk period $(n=223)$ & \\
\hline No & - & No & - \\
\hline Yes & $3.43(1.16-10.14)^{* *}$ & Yes & $3.46(1.17-10.24)^{* *}$ \\
\hline Ethnicity $(n=226)$ & & Ethnicity $(n=223)$ & \\
\hline White British & - & White British & - \\
\hline African-Caribbean & $1.50(0.70-3.22)$ & African-Caribbean & $1.32(0.61-2.87)$ \\
\hline Black African & $3.03(1.25-7.35)^{* *}$ & Black African & $2.77(1.15-6.65)^{* *}$ \\
\hline White other & $2.36(0.62-8.99)$ & White other & $2.31(0.60-8.80)$ \\
\hline Asian & $0.97(0.25-3.72)$ & Asian & $1.04(0.27-4.05)$ \\
\hline Other & $0.79(0.16-3.87)$ & Other & $0.38(0.05-3.18)$ \\
\hline \multicolumn{4}{|l|}{ Clinical } \\
\hline Diagnosis $(n=225)$ & & Diagnosis $(n=221)$ & \\
\hline Psychoses NOS & - & Brief psychotic disorder & - \\
\hline Delusional disorder & $1.98(0.60-6.51)$ & $\begin{array}{l}\text { Major depression with } \\
\text { psychotic features }\end{array}$ & $1.11(0.27-4.58)$ \\
\hline Acute and transient psychoses & $0.54(0.17-1.73)$ & $\begin{array}{l}\text { Bipolar disorder and mania with } \\
\text { psychotic features }\end{array}$ & $0.13(0.02-0.85)^{* *}$ \\
\hline Schizo-affective & $0.54(0.18-1.67)$ & Schizo-affective & $2.05(0.43-9.78)$ \\
\hline $\begin{array}{l}\text { Bipolar disorder and mania with } \\
\text { psychotic features }\end{array}$ & $0.04(0.01-0.21)^{* *}$ & Delusional disorder & $4.44(0.94-21.00)^{*}$ \\
\hline $\begin{array}{l}\text { Major depression with } \\
\text { psychotic features }\end{array}$ & $0.43(0.17-1.12)^{*}$ & Psychoses NOS & $2.55(0.58-11.18)$ \\
\hline $\begin{array}{l}\text { Schizotypal personality } \\
\text { disorder }\end{array}$ & - & Drug-induced psychoses & $0.78(0.14-4.24)$ \\
\hline Drug-induced psychoses & $0.19(0.05-0.80)^{* *}$ & $\begin{array}{l}\text { Other disorder } \\
\text { Unknown/unclear }\end{array}$ & - \\
\hline $\begin{array}{l}\text { Family history of psychosis } \\
(n=158)\end{array}$ & & $\begin{array}{l}\text { Family history of psychosis } \\
(n=157)\end{array}$ & \\
\hline No & - & No & - \\
\hline Yes & $1.07(0.47-2.46)$ & Yes & $1.39(0.61-3.16)$ \\
\hline Log DUP, days $(n=219)$ & $1.16(0.99-1.35)^{*}$ & Log DUP, days $(n=216)$ & $1.14(0.98-1.33)^{*}$ \\
\hline Log age of onset $(n=219)$ & $0.65(0.27-1.57)$ & Log age of onset $(n=216)$ & $0.51(0.21-1.27)$ \\
\hline Any drug use at baseline $(n=207)$ & & Any drug use at baseline $(n=204)$ & \\
\hline No use & - & No use & - \\
\hline Use & $0.83(0.45-1.56)$ & Use & $0.97(0.52-1.82)$ \\
\hline Mode of onset $(n=200)$ & & Mode of onset $(n=195)$ & \\
\hline Sudden & - & Sudden & - \\
\hline Acute & $0.63(0.25-1.57)$ & Acute & $0.56(0.22-1.42)$ \\
\hline Insidious & $1.32(0.61-2.86)$ & Insidious & $1.22(0.56-2.66)$ \\
\hline $\begin{array}{l}\text { Symptom dimension-log }(n=202) \\
\text { mania }\end{array}$ & $0.35(0.21-0.57)^{* *}$ & $\begin{array}{l}\text { Symptom dimension-log }(n=201) \\
\text { mania }\end{array}$ & $0.37(0.23-0.60)^{* *}$ \\
\hline $\begin{array}{l}\text { Symptom dimension-log }(n=202) \\
\text { reality distortion }\end{array}$ & $1.73(1.07-2.81)^{* *}$ & $\begin{array}{l}\text { Symptom dimension-log }(n=201) \\
\text { reality distortion }\end{array}$ & $1.77(1.08-2.91)^{* *}$ \\
\hline $\begin{array}{l}\text { Symptom dimension-log }(n=202) \\
\text { negative }\end{array}$ & $1.84(1.15-2.93)^{* *}$ & $\begin{array}{l}\text { Symptom dimension-log }(n=201) \\
\text { negative }\end{array}$ & $1.77(1.10-2.87)^{* *}$ \\
\hline
\end{tabular}


Table 5 (cont.)

\begin{tabular}{|c|c|c|c|}
\hline Predictor & $\begin{array}{l}\text { ICD unadjusted OR } \\
(95 \% \mathrm{CI})\end{array}$ & Predictor & $\begin{array}{l}\text { DSM unadjusted OR } \\
(95 \% \mathrm{CI})\end{array}$ \\
\hline $\begin{array}{l}\text { Symptom dimension-log }(n=202) \\
\text { depression }\end{array}$ & $1.03(0.68-1.56)$ & $\begin{array}{l}\text { Symptom dimension-log }(n=201) \\
\text { depression }\end{array}$ & $1.09(0.72-1.66)$ \\
\hline $\begin{array}{l}\text { Symptom dimension-log }(n=202) \\
\text { disorganization }\end{array}$ & $0.73(0.37-1.46)$ & $\begin{array}{l}\text { Symptom dimension-log }(n=201) \\
\text { disorganization }\end{array}$ & $0.70(0.35-1.41)$ \\
\hline \multicolumn{4}{|l|}{ Social } \\
\hline Living situation $(n=223)$ & & Living situation $(n=220)$ & \\
\hline Alone & - & Alone & - \\
\hline Not alone & $0.49(0.26-0.89)^{* *}$ & Not alone & $0.47(0.25-0.87)^{* *}$ \\
\hline Relationship status $(n=218)$ & & Relationship status $(n=215)$ & \\
\hline Single & - & Single & - \\
\hline Not single & $0.27(0.12-0.59)^{* *}$ & Not single & $0.28(0.13-0.61)^{* *}$ \\
\hline Highest education level $(n=220)$ & & Highest education level $(n=218)$ & \\
\hline School & - & School & - \\
\hline Further & $0.52(0.25-1.11)^{*}$ & Further & $0.46(0.21-0.99)^{* *}$ \\
\hline Higher & $0.61(0.25-1.53)$ & Higher & $0.60(0.24-1.50)$ \\
\hline Employment status $(n=218)$ & & Employment status $(n=215)$ & \\
\hline Unemployed & - & Unemployed & - \\
\hline Other & $0.55(0.28-1.06)^{*}$ & Other & $0.64(0.33-1.22)$ \\
\hline Contact with friends $(n=148)$ & & Contact with friends $(n=147)$ & \\
\hline Daily & - & Daily & - \\
\hline Weekly & $2.24(0.76-6.56)$ & Weekly & $1.88(0.67-5.28)$ \\
\hline Less than weekly & $4.49(1.54-13.05)^{* *}$ & Less than weekly & $3.50(1.24-9.85)^{* *}$ \\
\hline
\end{tabular}

ICD, International Classification of Diseases; DSM, Diagnostic and Statistical Manual of Mental Disorders; OR, odds ratio; CI, confidence interval; NOS, not otherwise specified; DUP, duration of undiagnosed psychosis.

${ }^{*} p<0.1,{ }^{* *} p<0.05$.

symptoms (OR 1.84, 95\% CI 1.15-2.93); living along (not living alone OR 0.49, 95\% CI 0.26-0.89); being single (not single OR $0.27,95 \%$ CI $0.12-0.59$ ); and having contact with friends less than weekly (OR 4.49, 95\% CI 1.54-13.05).

There was strong evidence that the following baseline variables were associated with not changing diagnosis to schizophrenia: having a diagnosis of bipolar disorder (OR 0.04, 95\% CI 0.01-0.21) or drug-induced psychoses (OR 0.19, 95\% CI 0.05-0.80); and having manic symptoms (OR 0.35, 95\% CI 0.21-0.57).

There was some weak evidence that the following were associated with changing to a diagnosis of schizophrenia: being male (female OR 0.57, 95\% CI 0.31-1.03); having psychotic major depression (OR $0.43,95 \%$ CI 0.17-1.12); being unemployed (other OR $0.55,95 \%$ CI $0.28-1.06$ ); and not being in further education (further education OR 0.52, 95\% CI 0.25-1.11).

For the DSM, there was some evidence that 13 baseline variables were associated with a change to schizophrenia. There was strong evidence that the following were associated with change to schizophrenia: being from London (Nottingham OR 0.34, 95\% CI 0.18-0.65); being male (female OR 0.50, 95\% CI 0.27-0.92); being in the age risk period (OR 3.46, 95\% CI 1.17-10.24); being black African (OR 2.77, 95\% CI 1.15-6.65); having a diagnosis of delusional disorder (OR 4.44, 95\% CI 0.94-21.00); having symptoms of reality distortion (OR 1.77, 95\% CI 1.08-2.91); having negative symptoms (OR 1.77, 95\% CI 1.10-2.87); living alone (not living alone OR $0.47,95 \%$ CI $0.25-0.87$ ); being single (not being single OR $0.28,95 \%$ CI $0.13-0.61$ ); not being in further education (further education OR $0.46,95 \%$ CI $0.21-$ 0.99); and having contact with friends less than weekly (OR 3.50, 95\% CI 1.24-9.85).

There was strong evidence that the following were associated with not changing diagnosis: having a diagnosis of bipolar disorder (OR 0.13 , 95\% CI 0.02-0.85); and having manic symptoms (OR 0.37, 95\% CI 0.23-0.60). There was some weak evidence that longer DUP was associated with changing diagnosis (OR 1.14, 95\% CI 0.98-1.33). For both the ICD and DSM, the numbers were too small to perform multivariate analyses.

\section{Discussion}

The prospective consistencies of both diagnostic systems were comparable at 55-60\%. Few demographic, 
clinical and social factors were associated with overall change in diagnosis but many factors were associated with change to schizophrenia in both DSM and ICD analyses. However, low numbers did not allow for multivariate analysis of variables associated with change to schizophrenia.

\section{Strengths and limitations}

As with most cohort studies, loss to follow-up is a potential bias. Not all incident cases could be given a lifetime diagnosis. However, there was no difference in the proportions followed up between the diagnoses and the overall prevalence of follow-up was respectable at $80 \%$. A further limitation was that missing data in the predictors of change analysis limited the power of the analyses. Despite these limitations, this study contributes evidence beyond previous research because of the incidence sample and 10-year follow-up using both the ICD and DSM diagnoses. Furthermore, it is based on consensus diagnoses made blind to ethnicity and baseline diagnoses and is the first study to examine so many potential predictors of diagnostic change across a range of domains (demographic, clinical and social).

\section{Findings and implications}

An important finding was that schizophrenia, schizoaffective disorder, bipolar disorder and drug-induced psychosis had higher numbers at follow-up, indicating a tendency for other diagnoses to migrate to these categories. This means that the incidence of these diagnoses may be underestimated in incidence studies and this may confuse the aetiological picture.

The prospective consistencies for schizophrenia, bipolar disorder and drug-induced psychosis were significantly higher than the overall stability $(>70 \%)$. The high stability of drug-induced psychosis is surprising. This category represents a specific subgroup of individuals who demonstrate a clear temporal relationship between onset and recovery of psychotic symptoms and substance use. Rates of co-morbid substance misuse are significant in first-episode psychosis cohorts at around 30-50\% (Cantwell et al. 1999; Van Mastrigt et al. 1999; Barnett et al. 2007), whereas drug-induced psychosis often represents less than $10 \%$ of a first-episode psychosis cohort (Whitty et al. 2005; Addington et al. 2006; Bromet et al. 2011). This suggests that often drug use is considered by assessors to be comorbid rather than a sole cause of first-episode psychosis but that where it is identified to be the sole cause, this is often correct.

Depressive psychosis shows lower prospective consistency $(45-50 \%)$. This might be expected given the anticipation that a substantial percentage of cases will develop bipolar affective disorder over time. However, approximately twice as many cases eventually receive a diagnosis of schizophrenia compared with bipolar disorder. Previous literature is consistent with this finding where consensus diagnosis was used over long periods of follow-up (Bromet et al. 2011). It is well recognized that there are prominent symptoms of both anxiety and depression in both prodromal and early psychosis (Birchwood et al. 2000) and this is further useful to clinicians considering treatment and prognosis.

The minimal crossover between the bipolar and schizophrenia categories illustrated in the movement matrix is further supported by the finding that a diagnosis of bipolar disorder is associated with reduced odds of changing diagnosis to schizophrenia over time. This taken with the propensity of depressive psychosis cases to change to schizophrenia suggests a complicated relationship between affective and 'so-called' non-affective psychosis.

The prospective and retrospective consistencies of the remaining categories were poor $(<40 \%$; with the exception of retrospective consistency for delusional disorder). Schizophreniform psychosis greatly overlaps with schizophrenia (duration of symptoms being the only difference) and explains the lower retrospective consistency of the DSM-IV-TR schizophrenia category, perhaps suggesting that it is an unnecessary extra category. Delusional disorder has significantly higher retrospective consistency (50-66\%), suggesting less crossover than the other unstable categories. The movement matrix showed more than half of cases with delusional disorder at baseline eventually receive a diagnosis of schizophrenia, and the ICD analysis revealed it is associated with changing diagnosis to schizophrenia. Given this, it may best be considered an attenuated form of schizophrenia.

Examination of the remaining categories in light of their instability - schizo-affective disorder, acute/brief psychosis and psychosis NOS - reveal no immediately obvious patterns or utility in terms of describing a course of symptoms. These categories represent approximately $15 \%$ of our sample (ICD 68 cases at baseline, 77 cases at follow-up; DSM-IV 64 cases at baseline, 63 cases at follow-up). This suggests that as well as lacking aetiologically driven diagnoses in psychiatry, for a significant minority of our patients we still lack an adequate descriptive framework, a challenge for new and future revisions of current classification systems.

\section{Change to diagnosis of schizophrenia}

While few characteristics were associated with diagnostic change in general, many factors were associated with change to schizophrenia in both DSM and ICD 
analyses. Perhaps unsurprisingly, these included variables associated with schizophrenia: symptoms of reality distortion, negative symptoms, and variables indicative of social isolation: living alone, being single and having contact with friends less than weekly. However, low numbers did not allow for multivariate analysis of variables associated with change to schizophrenia. This is in line with previous research that has reported negative symptoms (Mason et al. 1997) to be associated with a change in diagnosis to schizophrenia. The finding that social isolation (as measured by contact with friends) was associated with change to schizophrenia may be associated with negative symptoms, as less contact with friends could stem from negative symptoms, and lead to isolation (Schwartz et al. 2000).

\section{Conceptual considerations}

The findings from this and other studies on diagnostic stability raise two salient issues. The first is validity: there is an implicit assumption in papers on diagnosis that patients exhibiting stable diagnoses will demonstrate the same as-yet undiscovered physiological abnormalities. The finding that a number of diagnoses change over time is assumed to mean that the initial diagnosis was incorrect and thus the diagnostic systems are flawed, hence research into the causes of these conditions will be hampered. However, in the context of wider medicine, it is a common occurrence for diagnosis to change over time as a disorder develops, or as the clinical picture emerges. It is common that an acute condition precedes a chronic condition, for example, demyelination and multiple sclerosis. In this case, even information on aetiology and mechanism do not indicate outcome, as the mechanism for chronicity is separate. After an initial episode of this neuro-inflammatory disorder, there is little certainty as to prognosis - some people never relapse, for others further episodes occur and the diagnosis becomes multiple sclerosis (McDonald \& Compston, 2001). That acute cases of demyelination reoccur and can lead to a diagnosis of multiple sclerosis does not undermine the usefulness of this diagnosis.

This brings us to the second issue of clinical utility. It can be argued that diagnosis informs clinical management of the patient and is therefore useful. However, these results indicate that changes in diagnosis that would probably result in a change of management (e.g. from schizophrenia to bipolar disorder) are minimal, and the changes that do occur are less likely to result in a substantial change in management (e.g. delusional disorder to schizophrenia). Therefore, it could be argued that diagnosis is of little clinical relevance, and specific symptom change is the important thing to be aware of. However, knowledge of how these diagnoses might change over time may prove clinically useful for both doctors and patients in understanding prognosis early in the course of an illness, and, as ever, in quickly communicating the nature of a cluster of symptoms between clinicians.

This raises the question of whether we should be ignoring diagnosis, giving no prognosis to patients and families, and be treating based on symptoms rather than diagnosis. In some early intervention practices, it is now commonplace to assign a broad clinical diagnosis of psychosis early in the course of illness. This avoids using stigmatizing terms such as schizophrenia with its association with poor outcome, promoting the recovery model, and being optimistic about outcomes. It also acknowledges what we have demonstrated in the current study: early on in the course of psychotic illness, the precise diagnosis is often provisional. However, previous research suggests that diagnosis is very strongly associated with outcome (Hegarty et al. 1994), and thus not to share this with patients and their families could be unethical. Perhaps a better approach would be to acknowledge the provisional nature of initial diagnosis and be cautious in making inferences regarding prognosis based on diagnosis, as it may not reflect the long-term picture, and to be vigilant for changes in the clinical picture.

The stability of the current classification systems varies widely between diagnoses. Schizophrenia, bipolar disorder, drug-induced psychosis, delusional disorder and depressive psychosis all show relative stability and consistent patterns of change where change does occur. The findings on diagnostic change in depressive and bipolar cases poses a challenge to the distinction of affective/non-affective categories. Psychosis NOS, schizo-affective disorder and acute/brief psychosis perform poorly, yet still provide the best-fit diagnosis for $15 \%$ of our cohort, posing a significant challenge in the revision of these categories.

\section{Supplementary material}

For supplementary material accompanying this paper visit http://dx.doi.org/10.1017/S0033291715000720

\section{Acknowledgements}

This work was supported by the UK Medical Research Council (reference G0500817) and the Department of Health through the National Institute for Health Research (NIHR) Specialist Biomedical Research Centre for Mental Health award to the SLaM NHS Foundation Trust and the Institute of Psychiatry at King's College London. 


\section{Declaration of Interest}

R.M.M. has received speaker honoraria from Janssen, Astra-Zeneca, Lilly, BMS and Roche. P.B.J. is a member of scientific advisory boards for Roche and Otsuke. The other authors have nothing to disclose.

\section{References}

Aadamsoo K, Saluveer E, Kuunarpuu H, Vasar V, Maron E (2011). Diagnostic stability over 2 years in patients with acute and transient psychotic disorders. Nordic Journal of Psychiatry 65, 381-388.

Addington J, Chaves A, Addington D (2006). Diagnostic stability over one year in first-episode psychosis. Schizophrenia Research 86, 71-75.

American Psychiatric Association (2000). Diagnostic and Statistical Manual of Mental Disorders, Text Revision, 4th edn. (DSM-IV-TR). American Psychiatric Association:

Washington, DC.

Amin S, Singh SP, Brewin J, Jones PB, Medley I, Harrison G (1999). Diagnostic stability of first-episode psychosis: comparison of ICD-10 and DSM-III-R systems. British Journal of Psychiatry 175, 537-543.

Baca-Garcia E, Perez-Rodriguez MM, Basurte-Villamor I, Fernandez Del Moral AL, Jimenez-Arriero MA, Gonzalez De Rivera JL, Saiz-Ruiz J, Oquendo MA (2007a). Diagnostic stability of psychiatric disorders in clinical practice. British Journal of Psychiatry 190, 210-216.

Baca-Garcia E, Perez-Rodriguez MM, Basurte-Villamor I, Lopez-Castroman J, Fernandez Del Moral AL, Jimenez-Arriero MA, Gronzalez De Rivera JL, Saiz-Ruiz J, Leiva-Murillo JM, De Prado-Cumplido M, Santiago-Mozos R, Artes-Rodriguez A, Oquendo MA, De Leon J (2007b). Diagnostic stability and evolution of bipolar disorder in clinical practice: a prospective cohort study. Acta Psychiatrica Scandinavica 115, 473-480.

Baldwin P, Browne D, Scully PJ, Quinn JF, Morgan MG, Kinsella A, Owens JM, Russell V, O'Callaghan E, Waddington JL (2005). Epidemiology of first-episode psychosis: illustrating the challenges across diagnostic boundaries through the Cavan-Monaghan study at 8 years. Schizophrenia Bulletin 31, 624-638.

Barnett JH, Werners U, Secher SM, Hill KE, Brazil R, Masson K, Pernet DE, Kirkbride JB, Murray R, Bullmore ET, Jones PB (2007). Substance use in a population-based clinic sample of people with first-episode psychosis. British Journal of Psychiatry 190, 515-520.

Birchwood M, Iqbal Z, Chadwick P, Trower P (2000).

Cognitive approach to depression and suicidal thinking in psychosis. I: Ontogeny of post-psychotic depression. British Journal of Psychiatry 177, 516-528.

Bromet EJ, Kotov R, Fochmann LJ, Carlson GA, Tanenberg-Karant M, Ruggero C, Chang SW (2011). Diagnostic shifts during the decade following first admission for psychosis. American Journal of Psychiatry 168, 1186-1194.

Cantwell R, Brewin J, Glazebrook C, Dalkin T, Fox R, Medley I, Harrison G (1999). Prevalence of substance misuse in first-episode psychosis. British Journal of Psychiatry 174, 110-153.

Carr VJ, Lewin TJ, Neil AL, Halpin SA, Holmes S (2004). Premorbid, psychosocial and clinical predictors of the costs of schizophrenia and other psychoses. British Journal of Psychiatry 184, 517-525.

Craig T, Wanderling J, Janca A (2007). Long term diagnostic stability in international cohorts of persons with schizophrenia and related psychoses. In Prospects for Recovery From Schizophrenia-An International Investigation: Report From the WHO-Collaborative Project, The International Study of Schizophrenia (ed. K. Hopper, G. Harrison, A. Janca and N. Sartorius), pp. 50-60. Oxford University Press: Oxford.

Demjaha A, Morgan K, Morgan C, Landau S, Dean K, Reichenberg A, Sham P, Fearon P, Hutchington G, Jones PB, Murray RM, Dazzan P (2009). Combining dimensional and categorical representation of psychosis: the way forward for DSM-V and ICD-11? Psychological Medicine 39, 1943-1955.

Häfner H, Hambrecht M, Löffler W, Munk-Jørgensen P, Riecher-Rössler A (1998). Is schizophrenia a disorder of all ages? A comparison of first episodes and early course across the life-cycle. Psychological Medicine 28, 351-365.

Harrison G, Hopper K, Craig T, Laska E, Siegel C, Wanderling J, Dube KC, Ganey K, Giel R, an der Heiden W, Holmberg SK, Janca A, Lee PW, Leon CA, Malhotra S, Marsella AJ, Nakane Y, Sartorius N, Shen Y, Skoda C, Thara R, Tsirkin SJ, Varma VK, Walsh D, Wiersma D (2001). Recovery from psychotic illness: a 15- and 25-year international follow-up study. British Journal of Psychiatry 178, 506-517.

Hegarty JD, Baldessarini RJ, Tohen M, Waternaux C, Oepen G (1994). One hundred years of schizophrenia: a

metaanalysis of the outcome literature. American Journal of Psychiatry 151, 1409-1416.

Kirkbride JB, Fearon P, Morgan C, Dazzan P, Morgan K, Tarrant J, Lloyd T, Holloway J, Hutchinson G, Leff JP, Mallett RM, Harrison GL, Murray RM, Jones PB (2006). Heterogeneity in incidence rates of schizophrenia and other psychotic syndromes: findings from the 3-center ÆSOP study. Archives of General Psychiatry 63, 250-258.

Lane C (2013). Side effects: from quirky to serious, trends in psychology and psychiatry (http://www.psychologytoday. com/blog/sideeffects/201305/the-nimh-withdraws-supportdsm-5. 2013). Accessed September 2013.

Mason P, Harrison G, Croudace T, Glazebrook C, Medley I (1997). The predictive validity of a diagnosis of schizophrenia. A report from the International Study of Schizophrenia (ISoS) coordinated by the World Health Organization and the Department of Psychiatry, University of Nottingham. British Journal of Psychiatry 170, 321-327.

McDonald WI, Compston A (2001). Recommended diagnostic criteria for multiple sclerosis: guidelines from the International Panel on the Diagnosis of Multiple Sclerosis. Annals of Neurology 50, 121-127.

Morgan C, Lappin J, Heslin M, Donoghue K, Lomas B, Reininghaus U, Onyejiaka A, Croudace T, Jones PB, Murray RM, Fearon P, Doody GA, Dazzan P (2014). Reappraising the long-term course and outcome of psychotic disorders: The ÆSOP-10 Study. Psychological Medicine 44, 2713-2726. 
Robins E, Guze S (1970). Establishment of diagnostic validity in psychiatric illness: its application to schizophrenia. American Journal of Psychiatry 126, 983-987.

Sajith SG, Chandrasekaran R, Sadanandan Unni KE, Sahai A (2002). Acute polymorphic psychotic disorder: diagnostic stability over 3 years. Acta Psychiatrica Scandinavica 105, 104-109.

Salvatore P, Baldessarini RJ, Tohen M, Khalsa H-MK, Sanchez-Toledo JP, Zarate CA, Vieta E, Maggini C (2009). The McLean-Harvard First Episode Project: two-year stability of DSM-IV diagnoses in 500 first-episode psychotic disorder patients. Journal of Clinical Psychiatry 70, 458-466.

Salvatore P, Baldessarini RJ, Tohen M, Khalsa H-MK, Sanchez-Toledo JP, Zarate CA, Vieta E, Maggini C (2011). McLean-Harvard International First-Episode Project: two-year stability of ICD-10 diagnoses in 500 first-episode psychotic disorder patients. Journal of Clinical Psychiatry 72, 183-193.

Schimmelmann BG, Conus P, Edwards J, McGorry PD, Lambert M (2005). Diagnostic stability 18 months after treatment initiation for first-episode psychosis. Journal of Clinical Psychiatry 66, 1239-1246.

Schwartz JE, Fennig S, Tanenberg-Karant M, Carlson G, Craig T, Galambos N, Lavelle J, Bromet EJ (2000). Congruence of diagnoses 2 years after a first admission diagnosis of psychosis. Archives of General Psychiatry 57, 593-600.
StataCorp LP (2009). STATA 10.1 for Windows. StataCorp LP. College Station, TX.

Susser E, Finnerty M, Mojtabai R, Yale S, Conover S, Goetz R, Amador X (2000). Reliability of the Life Chart Schedule for assessment of the long-term course of schizophrenia. Schizophrenia Research 42, 67-77.

Van Mastrigt S, Addington J, Addington D (1999). Substance misuse at presentation to an early psychosis program. Social Psychiatry and Psychiatric Epidemiology 39, 69-72.

Veen ND, Selten JP, Schols D, Laan W, Hoek HW, van der Tweel I, Kahn RS (2004). Diagnostic stability in a Dutch psychosis incidence cohort. British Journal of Psychiatry 185, 460-464.

Whitty P, Clarke M, McTigue O, Browne S, Kamali M, Larkin C, O'Callaghan E (2005). Diagnostic stability four years after a first episode of psychosis. Psychiatric Services 56, 1084-1088.

World Health Organization (1993). The ICD-10 Classification of Mental and Behavioural Disorders: Diagnostic Criteria for Research. World Health Organization: Geneva.

World Health Organization (1994). Schedules for Clinical Assessment in Neuropsychiatry: Version 2 (SCAN V2). World Health Organization: Geneva.

World Health Organization (1996). Psychiatric and Personal History Schedule. World Health Organization: Geneva. 